BNL-73311-2004-IR

\title{
Sealed Combustion System with Diagnostic Self-tuning
}

\author{
C.R. Krishna
}

September 2004

Energy Resources Division, Energy Science and Technology Department

\section{Brookhaven National Laboratory \\ P.O. Box5000 \\ Upton, NY 11973-5000 \\ www.bnl.gov \\ Managed by \\ Brookhaven Science Associates, LLC}

for the United States Department of Energy under

Contract No. DE-AC02-98CH10886 


\section{DISCLAIMER}

This report was prepared as an account of work sponsored by an agency of the United States Government. Neither the United States Government nor any agency thereof, nor any of their employees, nor any of their contractors, subcontractors, or their employees, makes any warranty, express or implied, or assumes any legal liability or responsibility for the accuracy, completeness, or any third party's use or the results of such use of any information, apparatus, product, or process disclosed, or represents that its use would not infringe privately owned rights. Reference herein to any specific commercial product, process, or service by trade name, trademark, manufacturer, or otherwise, does not necessarily constitute or imply its endorsement, recommendation, or favoring by the United States Government or any agency thereof or its contractors or subcontractors. The views and opinions of authors expressed herein do not necessarily state or reflect those of the United States Government or any agency thereof: 
Sealed combustion system with Diagnostic Self-tuning

\subsection{Objective}

This task grew out of a proposal to develop a 'concept' oil-fired heating system that would incorporate advanced technologies available and suitable for a residential system. Discussions among the program and project personnel resulted in the identification of a sealed combustion system as of programmatic interest and the objective was to develop an approach to a sealed system with diagnostic self- tuning.

\subsection{Introduction}

This task was intended to examine the possibilities of developing, the sealed combustion system to be capable of modulation and of diagnostic selftuning. A 'sealed' system differs from a conventional system in that it is designed to draw the air for combustion from outside conditioned space and the venting system does not draw on air from the conditioned space either. This is typically done by enclosing the burner in a box or adding an air inlet boot to the burner, with a pipe supplying air to the box from outside the wall of the conditioned space. The hot gases from the heat exchanger are vented by using an inducer blower exhausting through the same wall as the intake. A modern commercially available oil-fired system that can be operated as a sealed system was chosen to conduct the experiments. The particular system, developed and marketed by Energy Kinetics (which uses a typical flame retention head burner), is like most if not all US marketed systems and has a pressure atomizer nozzle to which fuel is supplied at constant pressure. The fuel pump and the blower, which are traditionally part of the burner, are driven by the same constant speed motor, thus essentially ensuring a constant flow of both fuel and air under normal operation. Manual adjustment of the air inlet on the blower is available to change the airflows over a limited range to set the burner for different nozzle sizes and to allow for variability in installations and also because the burner is used by manufacturers of other boilers and furnaces. Thus, it was obvious that a modulating burner could not be achieved without fbndamental changes to the design of the burner (and possibly the boiler or heat exchanger) and while BNL has explored this in other projects, it was not considered feasible to explore in this task. Hence, the objective was modified to establishing the requirements for a control system to meet the requirements of a sealed combustion system, especially as it differs from a conventional 'non-sealed' system.

The key requirement in maintaining combustion performance is to keep the air fuel ratio constant on the assumption that the fbel spray and the airflow patterns are not changed. Deterioration of the fbel spray pattern due to partial blockage of the nozzle or pump pressure changes can cause undesirable changes in the flame. This may be, especially if the changes are severe, detected by change in the flame cad cell sensor operation and the control system shuts down the burner. This assumes that the components of the well-designed system such as the oil nozzle, and the blower (combustion air and the inducer) have not deteriorated. The Energy Kinetics system also has a 'puff switch' that responds to 
the pressure increase in the 'combustion chamber' and shuts the burner when this reaches a pre-set limit. Such pressure increase could occur if there is increased resistance to flow of the hot gases, caused for example by blockage in the heat exchanger or inducer failure. At the lower limit of air fuel ratio, typically the soot emission from the flame, as measured usually by the smoke number, starts increasing. At the higher limit in these 'fixed' systems, system malfunction is indicated. The usual way of detecting changes in air fuel ratio is to measure the oxygen in the exiting gases in the stack and compare it to the 'normal' value. This is the practice in larger stationary combustion systems, where the excess air is typically smaller than in smaller residential combustion systems. Usually, oxygen sensors are used to measure the oxygen level in the stack and the output from these are fed to the control system to perform the needed functions. This led to an assessment of whether such a sensor and measurement system could be used in the present case. In principle, it is possible to use an oxygen measurement to set a limit for the range of operation, that is shut the system down if the oxygen is lower than a preset limit for example. However, only one commercial oxygen sensor could be identified that was useable in the range of excess airs of such a system. This was a Honeywell sensor, currently offered by SST Sensing Ltd in UK and priced at $€ 346$ (Euros). This was deemed to be far too expensive for application to residential systems.

It was felt that pressure sensors could be inexpensive, as also indicated by the use of the 'puff switch' in the system, and hence the following approach to measure pressures in various parts of the system over the range of operation of interest was initiated in the hope of generating a control scheme based on one or two pressures at different locations. The results below show that the approach could be successful.

\subsection{Experimental Details}

Figure 1 is a photograph of the setup used in the tests. The sealed combustion system is an Energy Kinetics System 2000 boiler. The combustion air is drawn by the burner blower from the outside of the building wall through a PVC tube of 2 inches diameter. The hot exhaust gases leaving the heat exchanger of the boiler are exhausted through the same wall on which the intake is mounted by an inducer with an adjustable damper in its inlet. The damper is used to set the draft in the stack and in a residential installation is set to give the draft recommended in the system manual. The air shutter in the burner can be used to alter the amount of excess air and thus the oxygen level in the stack. Again, in a normal installation, it would be adjusted as recommended in the manual.

The key measurements in the tests were of pressures in the air and gas flow path. Initially, steady state static pressure measurements at 'static pressure' taps in the walls were made in the intake pipe, in the combustion chamber and in the stack. An MKS Baratron Type 170M-6C pressure meter with a pressure head of 10 Torr range was used for these measurements at steady state. The steady state readings were taken from the analog readout on the instrument. Following this, transient pressure measurements were made during start up and shut down at the same locations as for the steady state. The measurements were made over a short cycle of start up, steady run and shut down lasting usually less than a minute. The 
cycles were repeated at the same air shutter and stack damper settings for the measurements at the different locations and the air settings were nominally at the conditions recommended in the system manual. Three inducer damper positions were chosen as for the steady state tests to represent low, medium and high drafts on the outlet of the appliance. The transient data output fi-om the Baratron was recorded by a Tektronix TDS 420A digital oscilloscope. A sampling rate of 100 milliseconds was used in all the transient data acquisition.

There were several reasons for making the transient pressure measurements. Firstly, it is known that at ignition there is a positive (above ambient) pressure spike in the combustion chamber, even though the system design is such that under steady state conditions, the chamber pressure is maintained negative or below ambient. This is true for natural draft systems (chimney draft) and it is of interest to see how severe this is in a sealed system with induced draft. Secondly, if a pressure based control scheme is found to be feasible, it is necessary to know the length of the transient pressure pulse, as well as the fluctuations in steady state pressure for implementing a control scheme. Thirdly, there is an interest in data on the pressure spike, as it has potential to cause combustion gases to leak out of the combustion system. In a previous study at BNL, Butcher et al [1] had reviewed the previous measurements on transient behavior and modeled the pressure pulsations. They showed a startup peak of about 3 inches of water and damped sinusoidal pulsations lasting a little over 100 milliseconds. The suggestion made in the literature was that, if the blower/fan static pressure were higher than the startup peak, the pulsations would be damped more quickly than otherwise.

The magnitude of the pressure spike is a function of the ignition process, of the volume of gases which expand with the rapid temperature increase due to the flame and of the flow restriction due to the flue passage of the appliance including the vent. The System 2000 boiler has a relatively low operating pressure drop of .02 inches of water, but it does have a long spiral passage from the combustion area to the flue collar (See Figure 2).

The steady state tests were run in the usual way. Three different settings were chosen for the damper in front of the inducer fan and labeled, in an obvious way as low, medium and high draft. The air shutter settings were varied at each of the draft settings to obtain different excess air levels as measured by the oxygen concentration in the stack. Smoke measurements in the stack were made, as this is a key to the performance. Carbon monoxide levels remained low in all the tests.

Transient pressure measurements were made at three inducer positions, but at one air shutter position.. The burner was started, run up to steady state and shut down within a fairly short period of time and the pressure transducer outputs were captured during the entire period. 


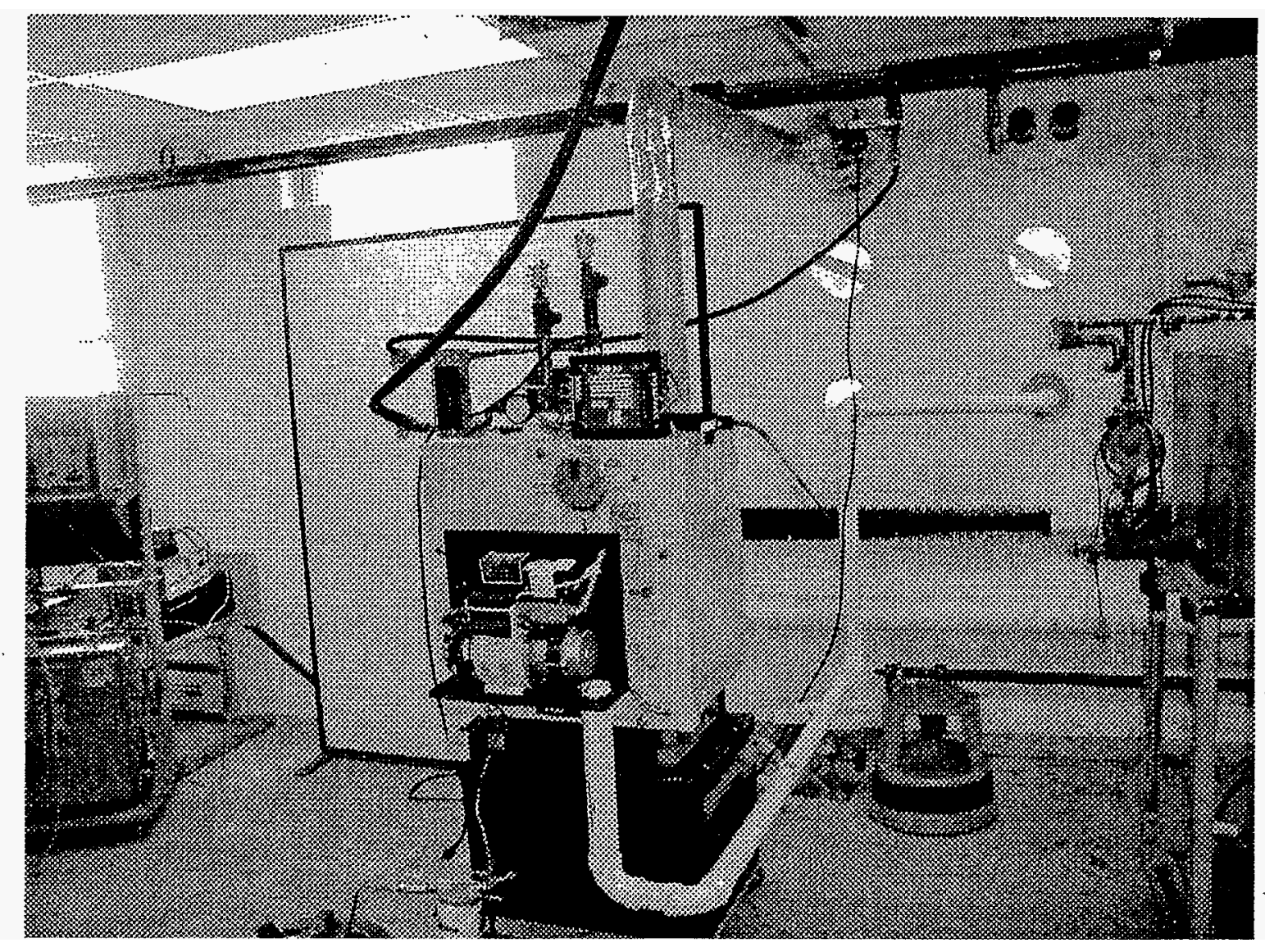

Figure 1. Photograph of Boiler set up 


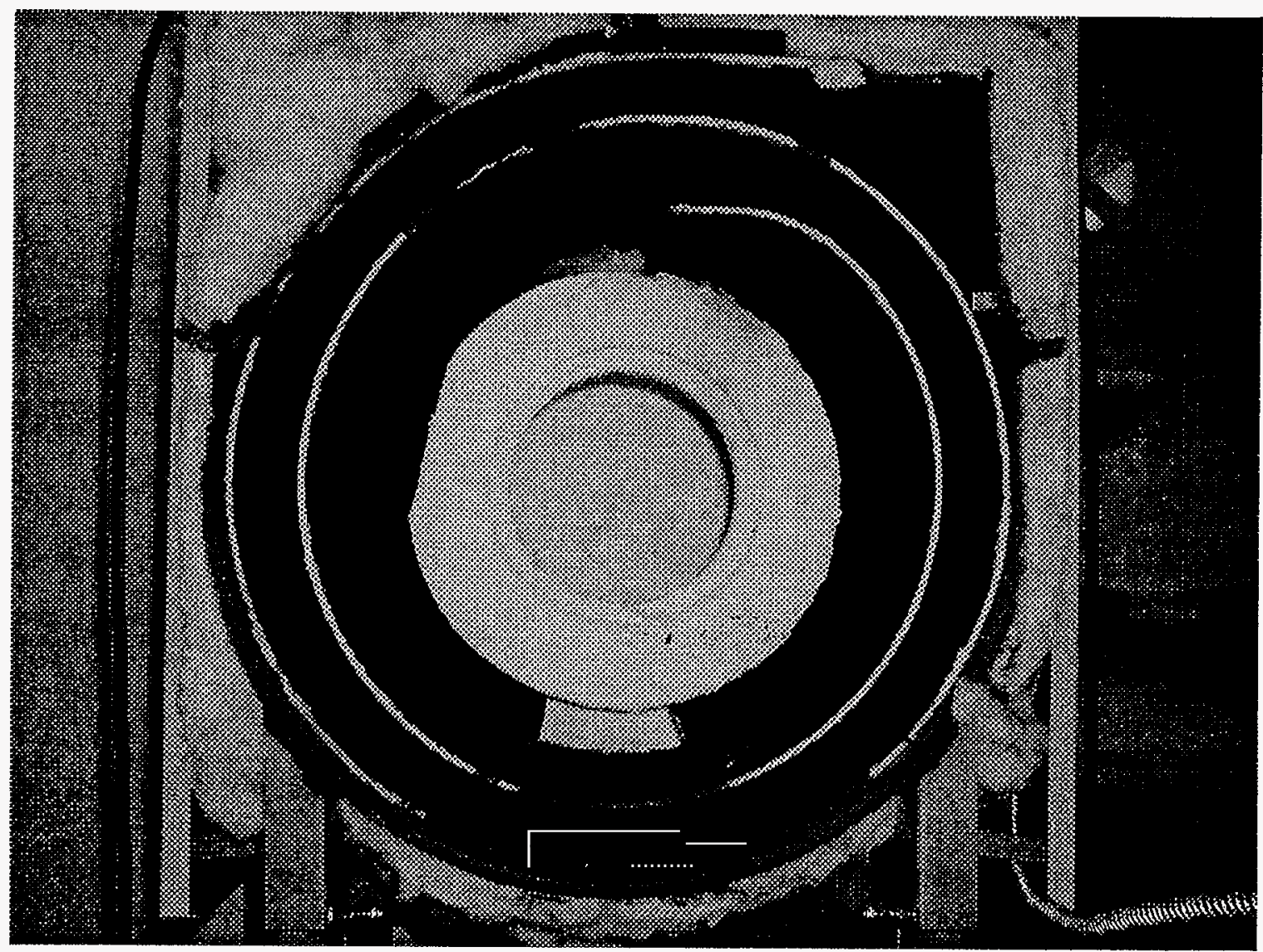

Figure 2. Spiral Passage

\subsection{Test Results and Discussion}

\section{A. Steady State Results}

Figure 3 below gives the basic performance of the boiler as normally represented by plotting the smoke number against the excess air given in terms of the stack oxygen. The three curves at low, medium, and high drafts were obtained by changing the settings of the damper in front of the inducer. These drafts span the recommended range in the manual and are felt to exceed the range that would be normally encountered. 
Smoke vs. Oxygen

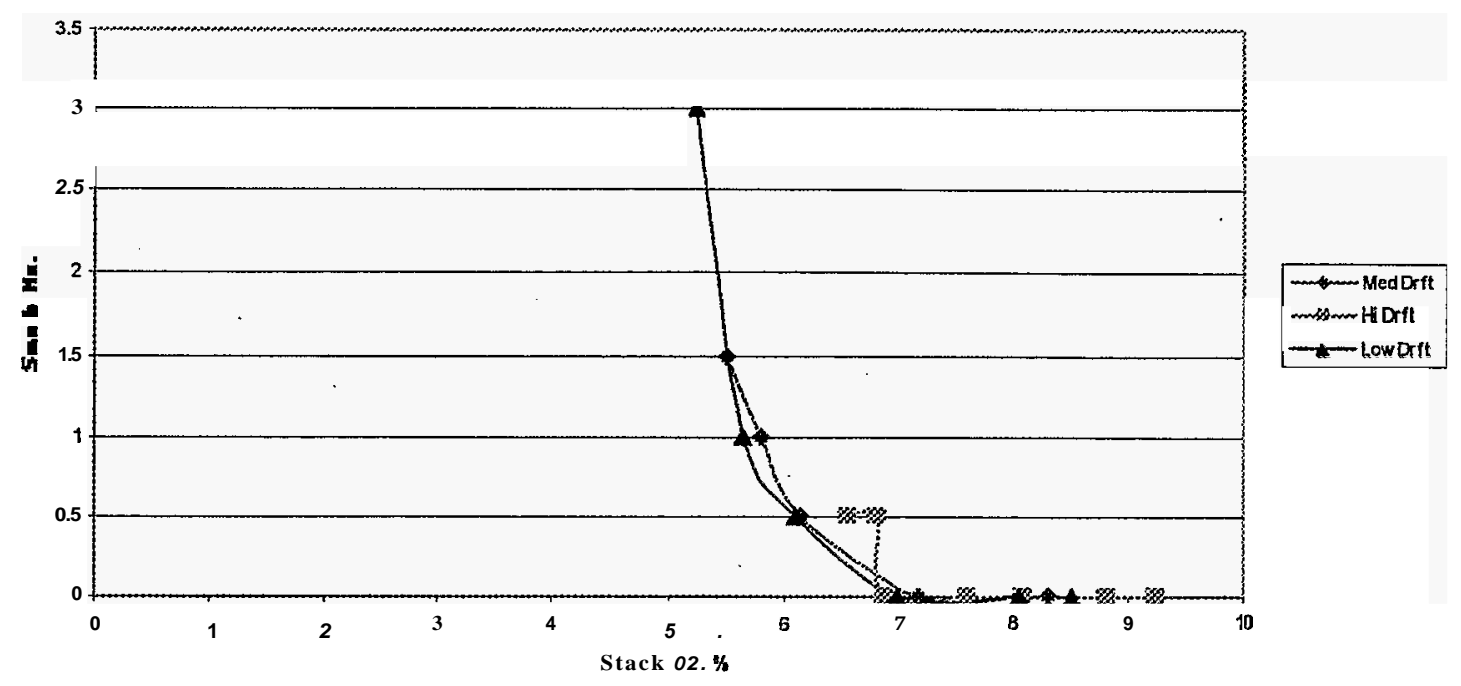

Figure 3. Boiler performance at different drafts

The main conclusion from this figure for the present purposes is that the smoke number tends to increase as the stack oxygen level drops below $7 \%$ for the range of drafts. This means, obviously, that this value could be used as a low limit in a limiting control. However, as indicated before, an oxygen sensor was found to be an expensive approach and hence we will attempt to extract from the pressure measurements below a similar unequivocal limiting value.

In the three figures 4, 5, and 6 below, three static pressures (gauge) are given as a function of the stack oxygen. They are respectively in the 'combustion' chamber, in the breech or stack, and in the intake pipe. It can be seen that the static pressures change more or less linearly with the stack oxygen content, though with an opposite slope for the intake in contrast with the other two. The lines are distinctly different for the different draft settings for the chamber and breech pressures. In contrast, the change with draft is much less for the intake pressure, so that one could represent reasonably the pressure-stack oxygen relationship with one line. This suggests strongly that the intake pressure could be the basis for setting an unequivocal range or limit for operation of the system at one firing rate. Analysis of similar measurements made at the other locations giving chamber pressures, stack pressures etc. as a function of excess air did not reveal any simple approach to finding a 'unique' control law. 


\section{Chamber Pressure}

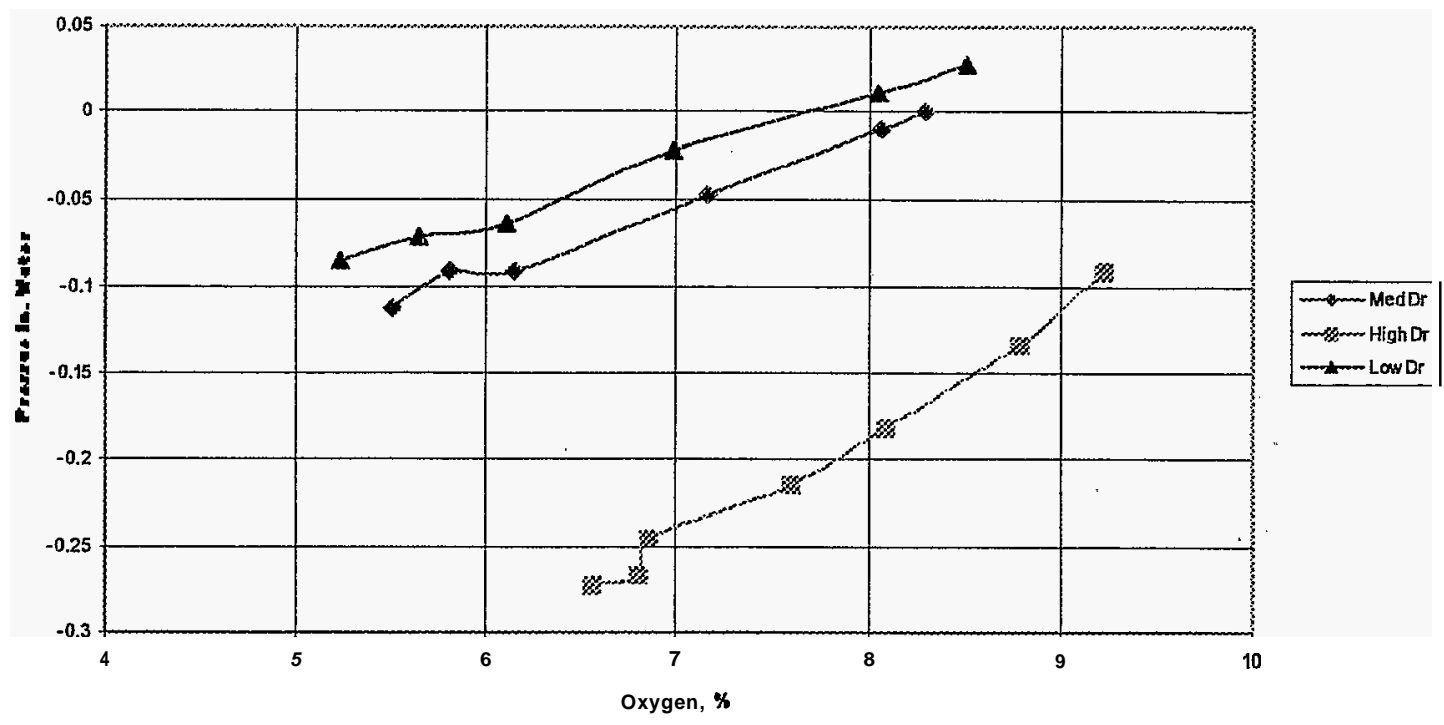

Figure 4. Static Pressures in Combustion Chamber

\section{Breech Pressure}

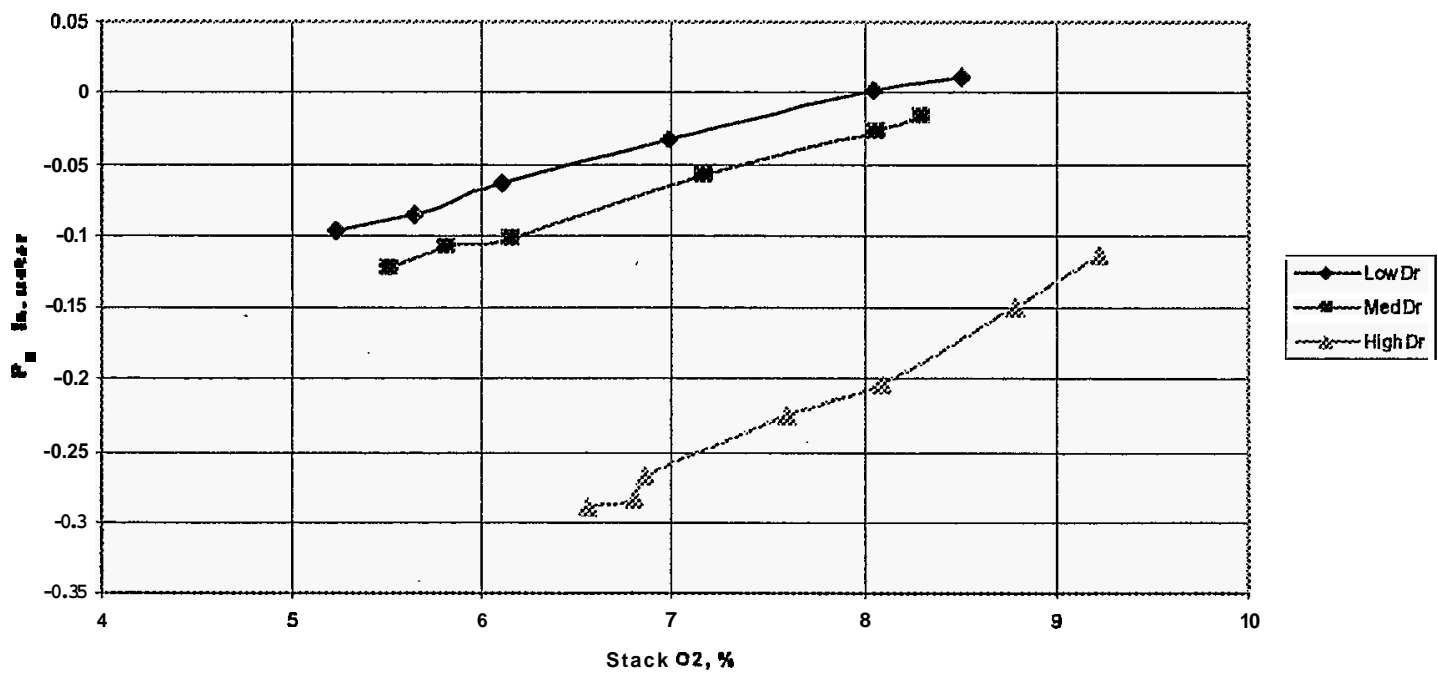

Figure 5. Static Pressures in the Stack 


\section{intake Pressure}

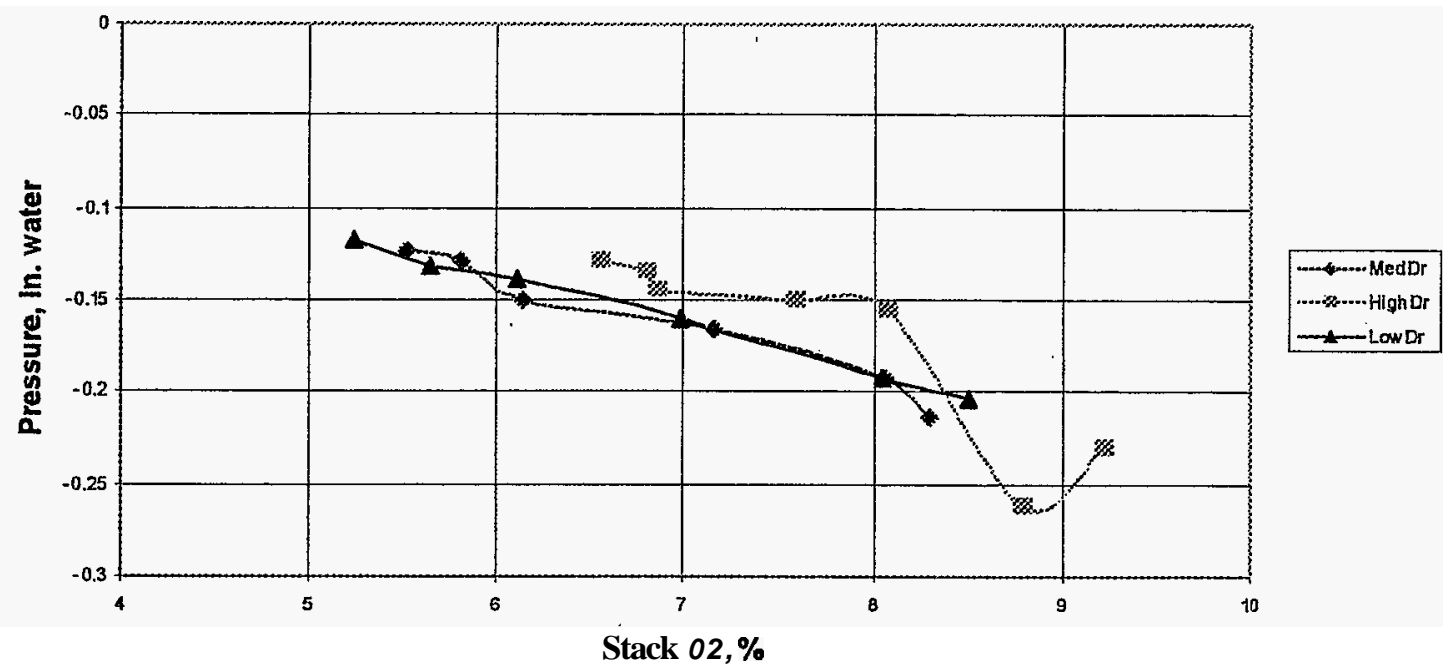

Figure 6. StaticPressures in the Intake

Smoke-Intake Pressure
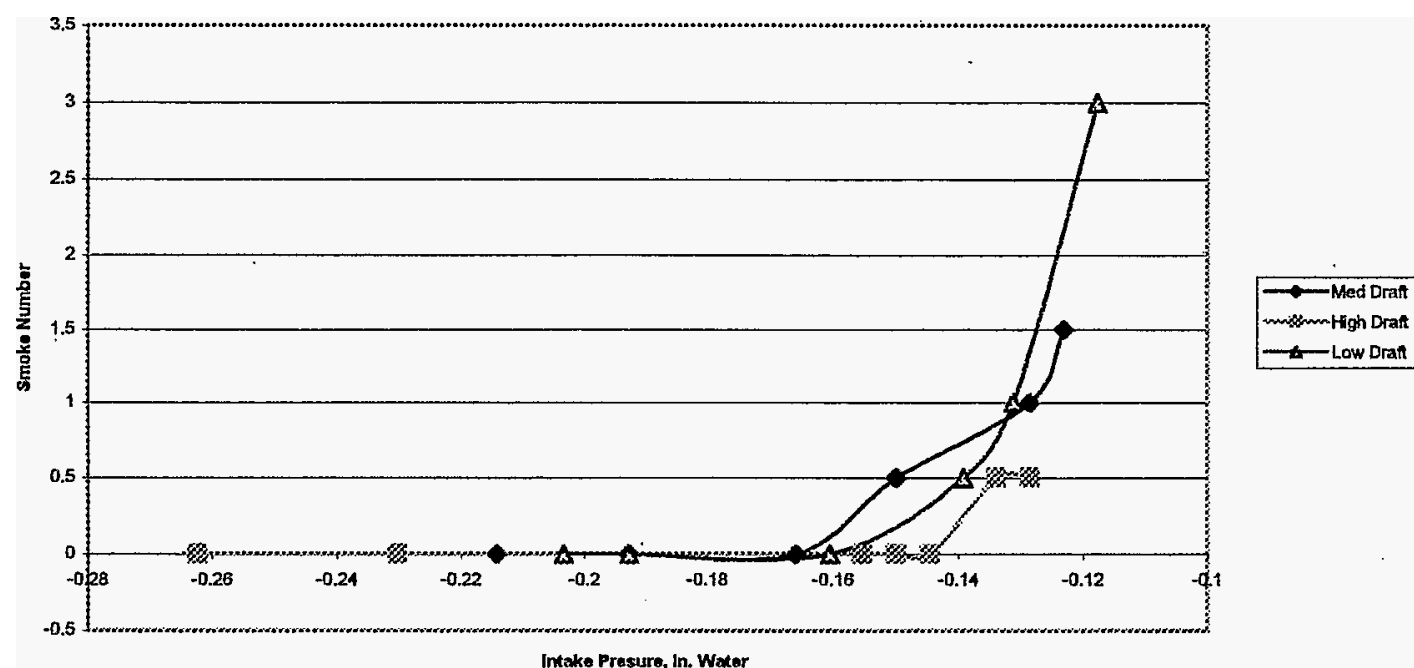

Figure 7. Smoke Number versus Intake Pressure

This conclusion is strengthened by Figure 7 above, which is a plot of the smoke numbers against the intake pressures. It would appear that limiting 
the intake pressure to a maximum pressure of about -0.17 inches of water would ensure that the system operates smoke free for this firing rate.

A little explanation of the behavior of the various pressures is relevant here. The chamber and breech pressures clearly increase with increase in the combustion airflow, represented by stack oxygen for a constant fuel input. Also, the values for the same airflow are dependent on the (constant) pressure increase through the combustion fan and the varying pressure increase with changing draft. The intake pressure is essentially a measure of the static pressure drop between the measurement location and the (constant) atmospheric pressure at the air intake outside the wall and thus is a measure of the airflow volume. Hence, it decreases with increase in airflow, nearly linearly and hence follows also the correlation with smoke number.

\section{B. Transient Test Results}

Figures 8, 9, and 10 below give the transient pressures in the combustion chamber at low, medium and high draft respectively. The 'jumps' in the pressure traces occur, starting from the left, at combustion fan start, ignition, and shut down. In all cases the startup pressure spike episode lasts for about a second or so. This is a function of the burner and ignition system providing a clean quick light off and possibly benefited by the ceramic combustion chamber. While the combustion lasts, there continues to be pressure fluctuations in this 'steady' operating condition, with the highest spikes into the positive pressure region at the lowest draft, as one would expect. These pressure fluctuations are obviously indicative of the combustion fluctuations. The other item to note is that there is a high negative pressure spike at shutdown of the same order as at startup and lasts a little bit longer. The magnitude of the startup pressure spike is about 0.6-0.7 inches of water in contrast to the calculated value of about 3 inches of water and literature measurements from 0.5 to 3 inches quoted in reference 1. The latter numbers are, presumably for non-sealed or conventional systems. It is possible that the use of the inducer leads to lower pressure spikes.

As the steady state measurements have suggested the use of the static pressure in the intake as the control parameter, it is useful to find how that pressure behaves in the transient conditions. Figures 11, 12, and 13 below show these measurements for the three draft conditions. The 'steady state' pressure shows fluctuations as in the chamber measurements but of a lesser magnitude. For the low and medium draft conditions, it is seen that the pressure spikes at startup are much lower, around 0.1 inch of water compared to about $0.6-0.7$ for the chamber. The negative pressures at shut down reach around 0.6 inches. However, the spike for the high draft at startup seems high at 0.6 inches unlike what one would expect. This might be an error in measurement or in transcription of data. In all cases however, the spikes seem to last a shorter time than in the chamber and for less than 400 milliseconds. Hence, the control sensor can be activated at a time several times this time length after startup (say a couple of seconds) and then should be able to provide the necessary input to the 
control for shut down. Diaphragm switches with orifices can probably provide this time sensitivity.

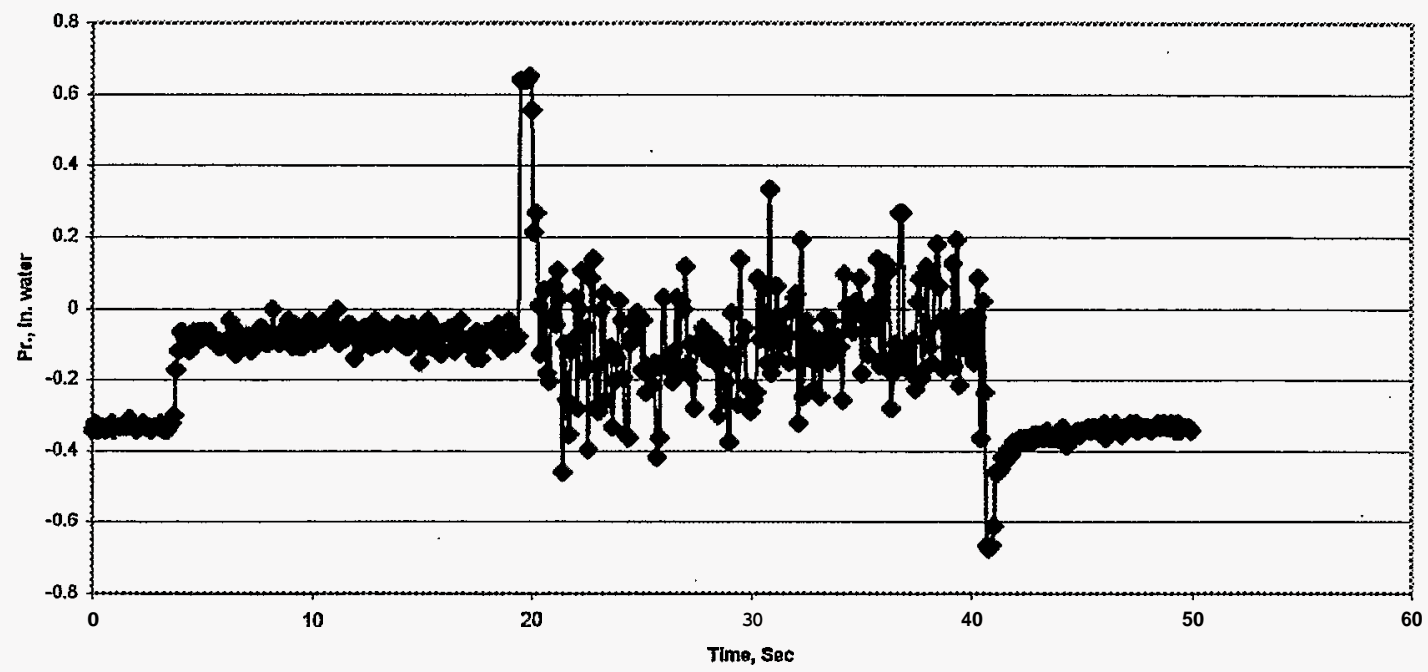

Figure 8. Transient pressures in the Combustion Chamber at low draft

\section{Chamber Transient Pressures at medium draft}

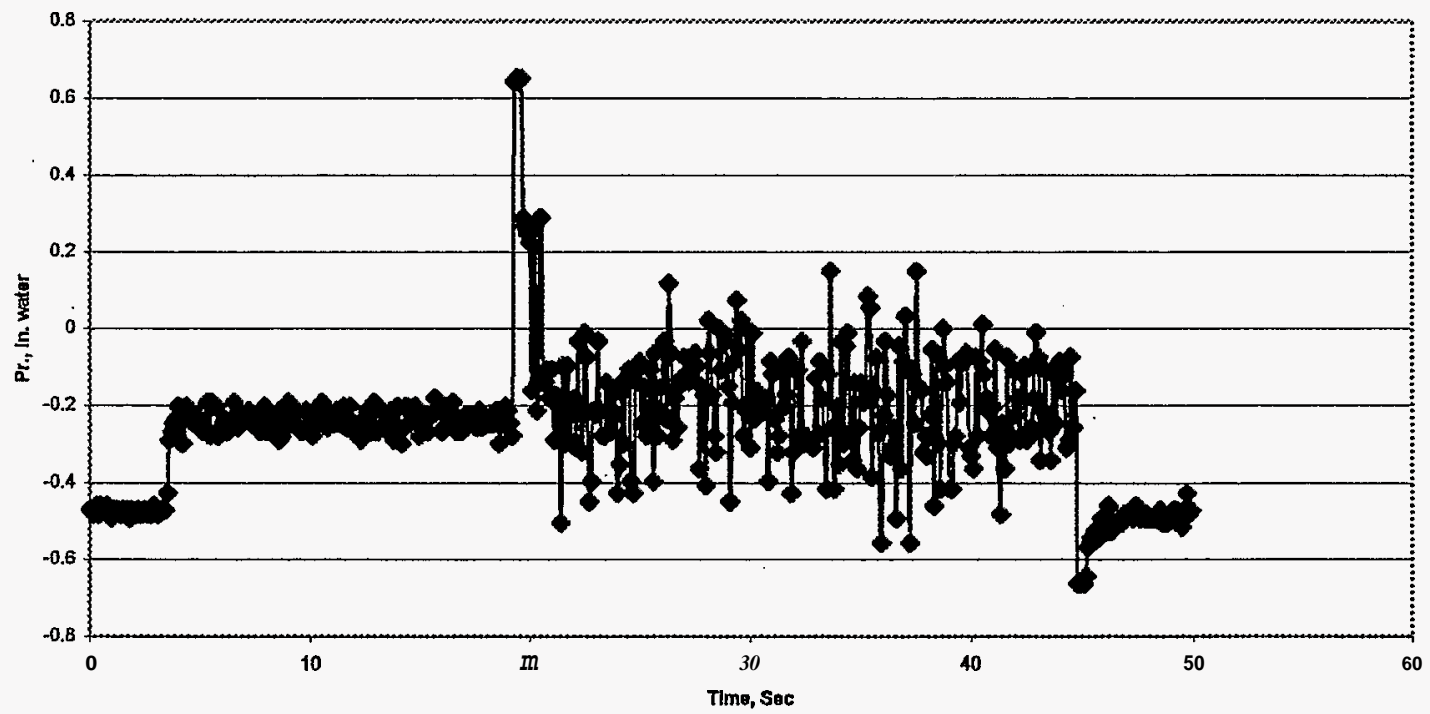

Figure 9. Transient pressures in the Combustion Chamber at medium draft 
Chamber Transient Pressures $\star$ High draft

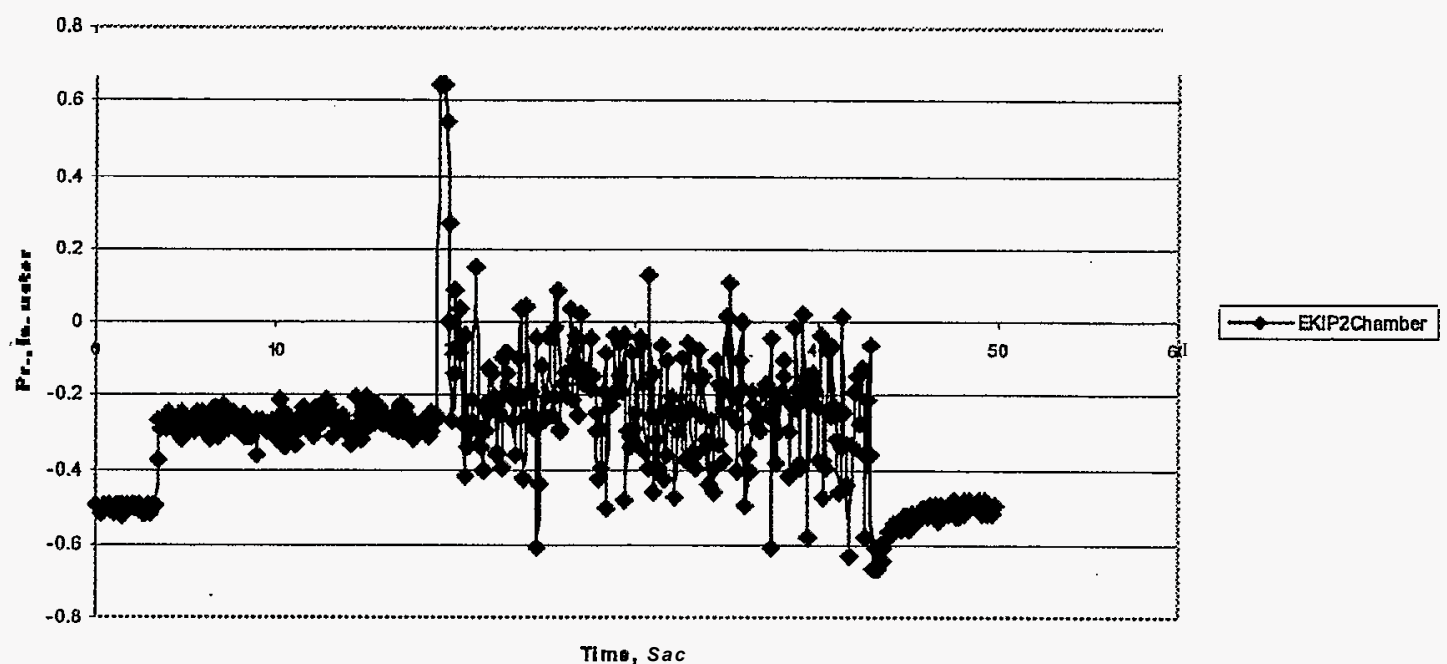

Figure 10. Transient pressures in the Combustion Chamber at high draft

Intake transient pressures

at low drat

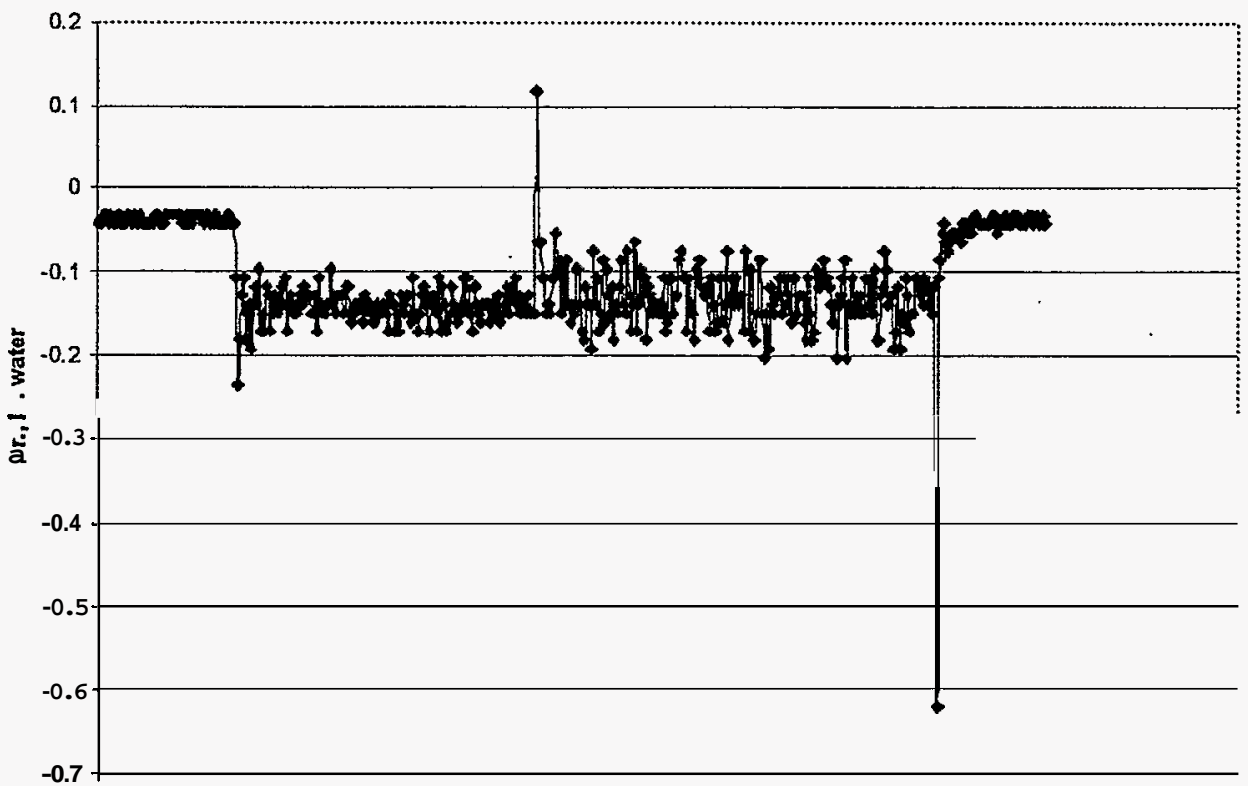


Intake Transient Pressures at mediumdraft

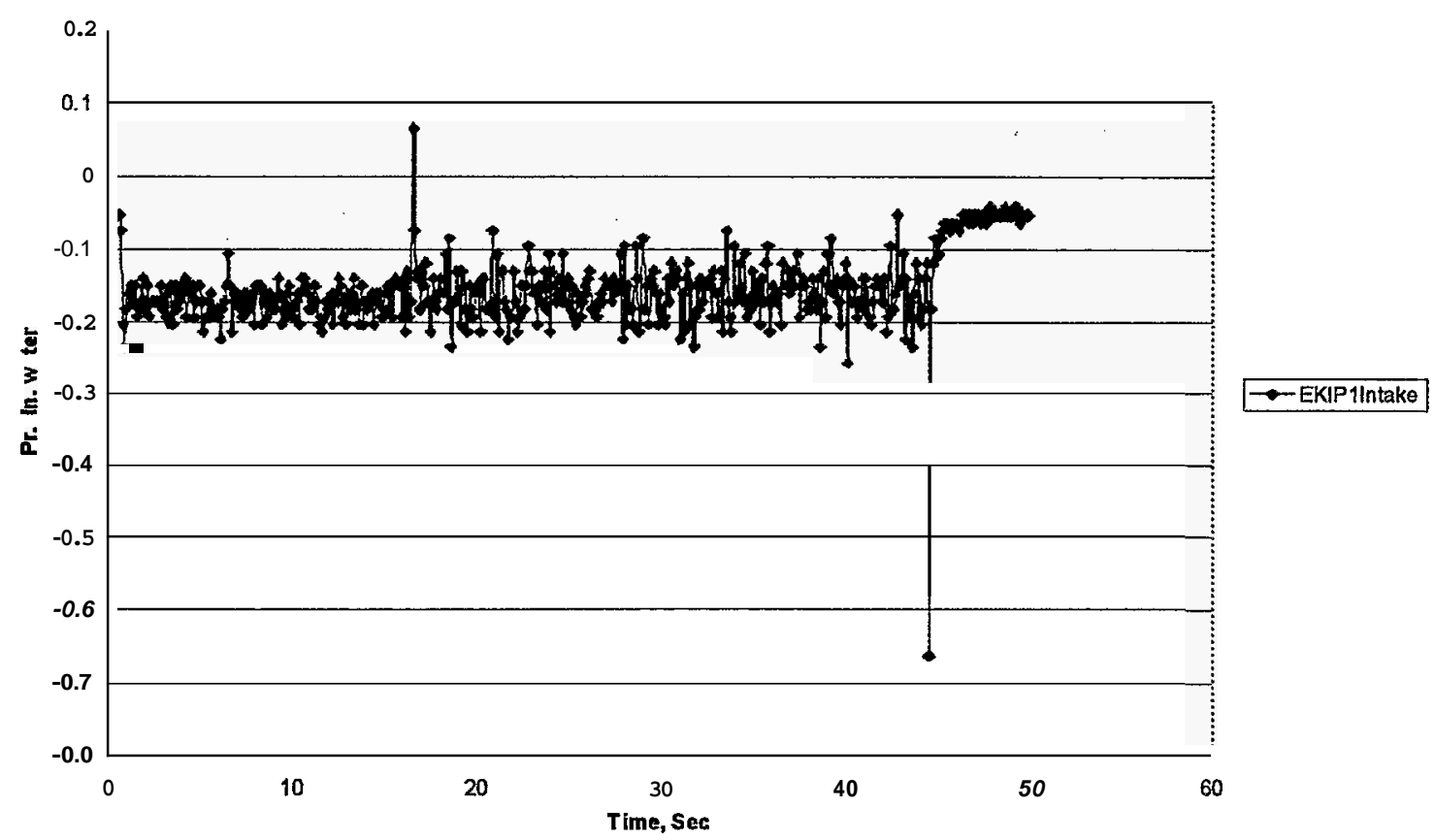

Figure 12. Transient pressures in the Intake at medium draft

Intake Transient Pressures

at high draft

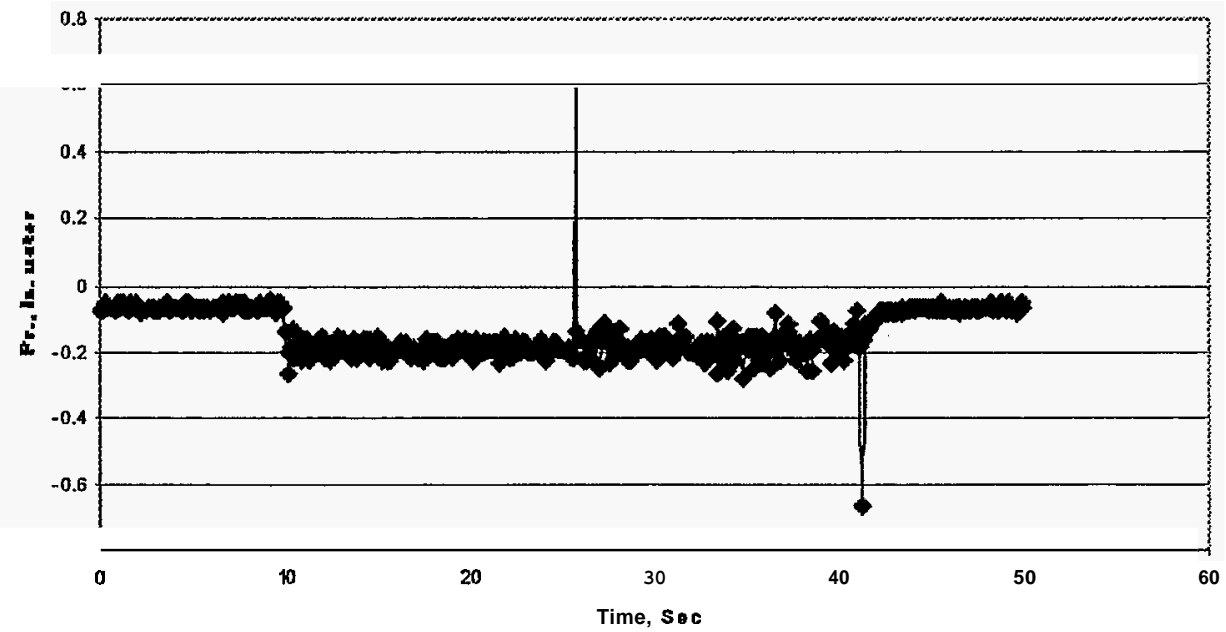

Figure 13. Transient Pressures in the Intake for high draft 


\subsection{Conclusions and Recommendations}

The major conclusion with regard to the specific objective for this task is that a measurement of the static pressure in the input pipe can be used to provide a measure of the excess air for a limit control. Its implementation would require a suitable pressure sensor, and a circuit to combine its output signal, at the appropriate time in the operating cycle, to the burner control. The sensor and control combination will also have to be tested successfully under all conceivable contingencies that can cause the airflow to decrease. It could also be implemented, possibly even more reliably, if a mass flow sensor, such as is used in automobile engines and hence may be cheap, could be used.

The pressure measurements reported here, both steady and transient, represent only the subset of a much larger set that gave a useful answer to meet the objective. These measurements suggest that they can provide useful insights into both the combustion and gas flow performance of the system. Of course, this could be extended to other types of heating systems, such as those with natural draft, those with different burners, burners with different atomization schemes (air atomization etc.), blue flame burners etc.

\subsection{References}

1. T. Butcher, F. McNeill, Y. Celebi, and J. Wegrzyn Impact of burner Design on Sooting in Residential Oil Fired Systems, BNL 52102 (1986). 